\title{
Multi-Lepton Production in ep Collisions at H1
}

\author{
Gerhard Brandt * \\ Universität Heidelberg - Physikalisches Institut \\ Philosophenweg 12, 69120 Heidelberg - Germany
}

\begin{abstract}
A search for multi-lepton (electron or muon) events at high transverse momenta in the data sample of $e^{ \pm} p$ collisions collected with the H1 detector at HERA in the years 1994-2007 is presented [1]. The data sample corresponds to an integrated luminosity of $459 \mathrm{pb}^{-1}$. Yields of di-lepton and tri-lepton events are measured and a general good agreement is found with Standard Model (SM) predictions. Combining all channels, four events are observed with a scalar sum of lepton transverse momenta $\left(\sum P_{T}\right)$ greater than $100 \mathrm{GeV}$, compared to a SM expectation of $1.9 \pm 0.4$.
\end{abstract}

\section{Introduction}

Multi-lepton events at high transverse momenta are of special interest as these signatures might reveal new physics beyond the SM. Events with two or more leptons with high transverse momentum $P_{T}$ are measured in electron-proton collisions at HERA using the full HERA I+II high-energy data sample at centre-of-mass energies up to $\sqrt{s}=320 \mathrm{GeV}$. The data were collected in the years 1994-2007 and correspond to an integrated luminosity of $459 \mathrm{pb}^{-1}$. This comprises of $286 \mathrm{pb}^{-1}$ of $e^{+} p$ collisions and $173 \mathrm{pb}^{-1}$ of $e^{-} p$ collisions.

Within the SM the production of multi-lepton events in $e p$ collisions mainly proceeds via photon-photon interactions [2]. Precise cross-section measurements of both electron (e) and muon $(\mu)$ pair production at high $P_{T}$ have already been performed by the $\mathrm{H} 1$ collaboration using HERA I data $[3,4]$.

\section{Selection}

Electron candidates are identified as compact clusters in the calorimeters with energies above $5 \mathrm{GeV}$ and in the polar angular range $5^{\circ}<\theta<175^{\circ}$. They are required to be isolated from other leptons or jets. In the central region $20^{\circ}<\theta<150^{\circ}$ the electron identification is complemented by tracking conditions, requiring a tight geometrical and $P_{T}$ match between track and cluster.

Muon candidates are identified with $P_{T}>2 \mathrm{GeV}$ in the range $20^{\circ}<\theta<160^{\circ}$ by associating a track in the inner tracking systems with a track segment or an energy deposit in the outer muon chambers. Calorimetric energy depositions in the vicinity of the track are required to be typical for minimally ionising particles, such as muons. In di-muon events, cosmic background radiation is reduced by the requirement that the opening angle between the two muons is smaller than $160^{\circ}$.

The final multi-lepton selection requires that there be at least two lepton candidates (electron or muon) identified in the central region, of which one must have $P_{T}>10 \mathrm{GeV}$ and the other $P_{T}>5 \mathrm{GeV}$. Additional identified lepton candidates are accepted without extra requirements. Leptons are counted and the events are classified into di-lepton $e e, \mu \mu$ and $e \mu$ samples, and tri-lepton $e e e$ and $e \mu \mu$ samples.

\footnotetext{
*for the H1 Collaboration.
} 
H1 Multi-lepton analysis HERA I+II $\left(459 \mathrm{pb}^{-1}\right)$
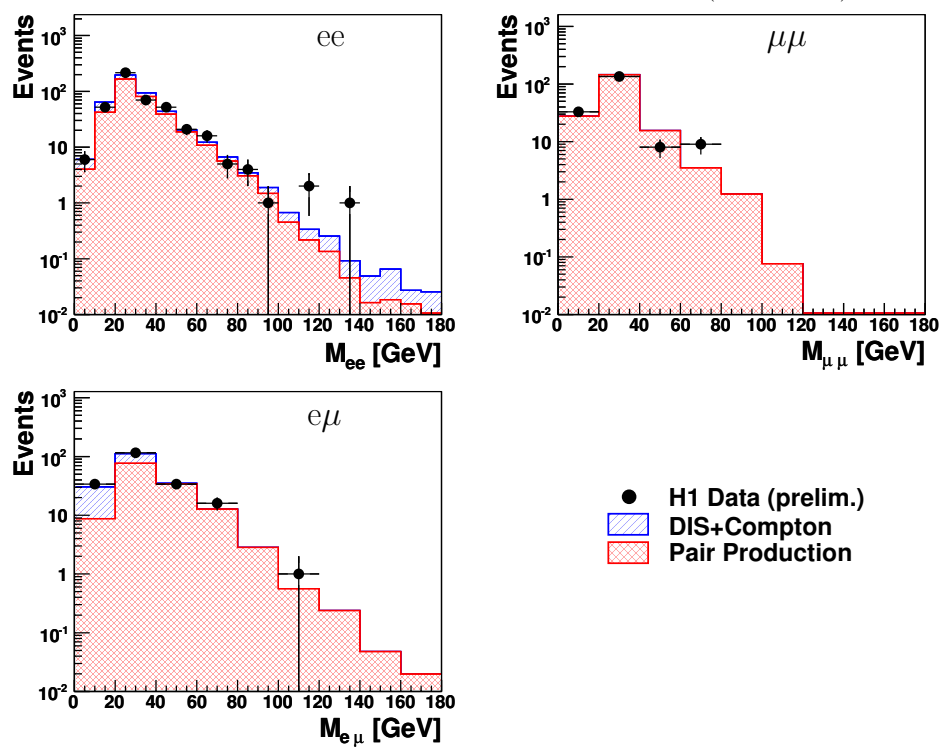

- H1 Data (prelim.)

DIS+Compton

Pair Production

Figure 1: Invariant masses $M_{\ell \ell^{\prime}}$ of the leptons for events selected in the di-lepton (ee, $\mu \mu$ and $e \mu$ ) classes compared to the SM expectation.

\section{Results}

The invariant mass $M_{\ell \ell^{\prime}}$ distributions for the two leptons in the di-lepton classes are shown in Fig. 1. The invariant mass distributions of the two highest $P_{T}$ leptons in the eee class $M_{12}$, and for the $e e \mu$ class the highest $P_{T}$ muon with the electron $M_{e \mu}$ and the second muon $M_{\mu \mu}$ are shown in Fig. 2. The observed event yields are in good agreement with the SM expectation which is dominated by pair production.

The event yields for all classes in the region $M_{\ell \ell^{\prime}}>100 \mathrm{GeV}$ are shown in Tab. 1, also for collisions with a positron or electron beam separately. The interesting events seen at high invariant masses in the ee and eee classes were observed in HERA I $e^{+} p$ data and previously reported [3]. Two e $\mu \mu$ events are observed in the new HERA II data, one of which has a high $\mu-\mu$ and the other a high $e-\mu$ invariant mass.

The event yields for events with $\sum P_{T}>100 \mathrm{GeV}$ are shown in Tab. 2. Four events are observed in all channels combined while $1.9 \pm 0.4$ are expected in the SM. These four data events correspond to the three ee events observed in HERA I data [3] and one new e $\mu \mu$ event observed in HERA II data. The four events with $\sum P_{T}>100 \mathrm{GeV}$ are all observed in $e^{+} p$ collisions only, where the SM expectation is $1.2 \pm 0.2$ events.

\section{Conclusions}

Multi-lepton events with electrons and muons were studied using the full HERA I+II data set. The measurement extends previous analyses by including HERA II data. The event yields in the di-lepton $(e e, \mu \mu$ and $e \mu$ ) and tri-lepton (eee and $e \mu \mu)$ sub-samples are in 
H1 Multi-lepton analysis HERA I+II $\left(459 \mathrm{pb}^{-1}\right)$
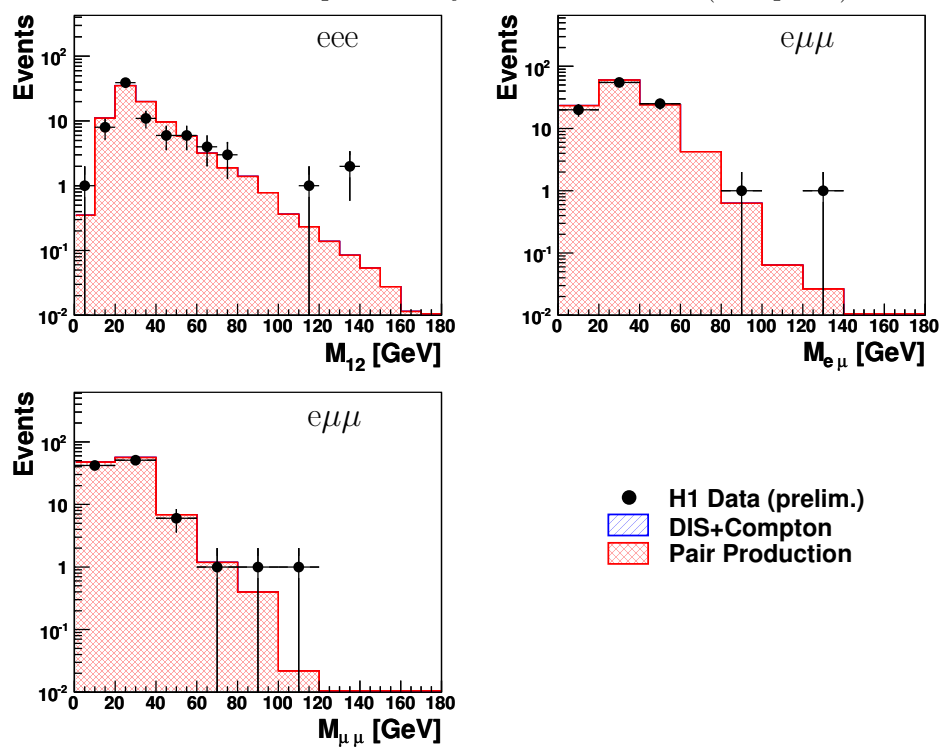

Figure 2: Distribution of the invariant mass $M_{12}$ of the two highest $P_{T}$ electrons for the eee sample (top left). For the e $\mu \mu$ sample, invariant mass combinations of the electron with the higest $P_{T}$ muon $\left(M_{e \mu}\right.$, top right) and of both muons $\left(M_{\mu \mu}\right.$, bottom left) are presented.

\begin{tabular}{|c|c|c|c|c|}
\hline Selection & Data & SM & Pair Production & NC-DIS + Compton \\
\hline \multicolumn{5}{|c|}{$e^{+} p$ collisions $\left(286 \mathrm{pb}^{-1}\right)$} \\
\hline ee $M_{12}>100 \mathrm{GeV}$ & 3 & $1.0 \pm 0.2$ & $0.6 \pm 0.2$ & $0.4 \pm 0.1$ \\
\hline$\mu \mu M_{\mu \mu}>100 \mathrm{GeV}$ & 0 & $0.06 \pm 0.03$ & $0.06 \pm 0.03$ & - \\
\hline $\mathrm{e} \mu M_{e \mu}>100 \mathrm{GeV}$ & 1 & $0.53 \pm 0.05$ & $0.53 \pm 0.05$ & - \\
\hline eee $M_{12}>100 \mathrm{GeV}$ & 3 & $0.6 \pm 0.1$ & $0.6 \pm 0.1$ & - \\
\hline $\mathrm{e} \mu \mu M_{e \mu}>100 \mathrm{GeV}$ & 1 & $0.04 \pm 0.02$ & $0.04 \pm 0.02$ & - \\
\hline $\mathrm{e} \mu \mu M_{\mu \mu}>100 \mathrm{GeV}$ & 1 & $0.007 \pm 0.005$ & $0.007 \pm 0.005$ & - \\
\hline \multicolumn{5}{|c|}{$e^{-} p$ collisions $\left(173 \mathrm{pb}^{-1}\right)$} \\
\hline ee $M_{12}>100 \mathrm{GeV}$ & 0 & $0.55 \pm 0.1$ & $0.3 \pm 0.1$ & $0.25 \pm 0.07$ \\
\hline$\mu \mu M_{\mu \mu}>100 \mathrm{GeV}$ & 0 & $0.03 \pm 0.02$ & $0.03 \pm 0.02$ & - \\
\hline $\mathrm{e} \mu M_{e \mu}>100 \mathrm{GeV}$ & 0 & $0.3 \pm 0.05$ & $0.3 \pm 0.05$ & - \\
\hline eee $M_{12}>100 \mathrm{GeV}$ & $\overline{0}$ & $0.32 \pm 0.06$ & $0.32 \pm 0.06$ & - \\
\hline $\mathrm{e} \mu \mu M_{e \mu}>100 \mathrm{GeV}$ & 0 & $0.04 \pm 0.01$ & $0.04 \pm 0.01$ & - \\
\hline $\mathrm{e} \mu \mu M_{\mu \mu}>100 \mathrm{GeV}$ & 0 & $0.006 \pm 0.004$ & $0.006 \pm 0.004$ & - \\
\hline \multicolumn{5}{|c|}{ All data $\left(459 \mathrm{pb}^{-1}\right)$} \\
\hline ee $M_{12}>100 \mathrm{GeV}$ & 3 & $1.5 \pm 0.3$ & $0.9 \pm 0.2$ & $0.6 \pm 0.2$ \\
\hline$\mu \mu M_{\mu \mu}>100 \mathrm{GeV}$ & 0 & $0.09 \pm 0.05$ & $0.09 \pm 0.05$ & - \\
\hline $\mathrm{e} \mu M_{e \mu}>100 \mathrm{GeV}$ & 1 & $0.9 \pm 0.1$ & $0.9 \pm 0.1$ & - \\
\hline eee $M_{12}>100 \mathrm{GeV}$ & 3 & $0.9 \pm 0.2$ & $0.9 \pm 0.2$ & - \\
\hline $\mathrm{e} \mu \mu M_{e \mu}>100 \mathrm{GeV}$ & 1 & $0.1 \pm 0.04$ & $0.1 \pm 0.04$ & - \\
\hline $\mathrm{e} \mu \mu M_{\mu \mu}>100 \mathrm{GeV}$ & 1 & $0.03 \pm 0.02$ & $0.03 \pm 0.02$ & - \\
\hline
\end{tabular}

Table 1: Yields for high di-lepton masses, $M>100 \mathrm{GeV}$ in all analysed samples. For the eee sample, the mass of the two highest $P_{T}$ electrons is shown. The errors on the prediction include model uncertainties and experimental systematic errors added in quadrature. 
H1 Multi-lepton analysis HERA I+II (459 $\mathrm{pb}^{-1}$, preliminary)

\begin{tabular}{|c|c|c|c|c|}
\hline \multicolumn{5}{|c|}{$\Sigma E_{T}>100 \mathrm{GeV}$} \\
\hline Data sample & Data & SM & Pair Production & NC-DIS + Compton \\
\hline $\mathrm{e}^{+} \mathrm{p}\left(286 \mathrm{pb}^{-1}\right)$ & 4 & $1.2 \pm 0.2$ & $1.0 \pm 0.2$ & $0.2 \pm 0.1$ \\
$\mathrm{e}^{-} \mathrm{p}\left(173 \mathrm{pb}^{-1}\right)$ & 0 & $0.8 \pm 0.2$ & $0.6 \pm 0.2$ & $0.2 \pm 0.1$ \\
All $\left(459 \mathrm{pb}^{-1}\right)$ & 4 & $1.9 \pm 0.4$ & $1.5 \pm 0.3$ & $0.4 \pm 0.1$ \\
\hline
\end{tabular}

Table 2: Yields of events with $\sum P_{T}>100 \mathrm{GeV}$ for the combination of di- and tri-leptons. The errors on the prediction include model uncertainties and experimental systematic errors added in quadrature.

good agreement with the SM prediction. The distribution of the scalar sum of transverse momenta of the leptons is studied for the combination of all di- and tri-lepton sub-samples. The overall agreement with the SM is good, but four interesting events with a large scalar sum of transverse momenta are observed in $e^{+} p$ collisions only, in a region where the SM expectation is $1.2 \pm 0.2$.

\section{References}

[1] Slides:

http://indico. cern. ch/contributionDisplay . py? contribId=126\&sessionId=9\&conf Id=9499 H1prelim-07-062:

http://www-h1.desy.de/h1/www/publications/htmlsplit/H1prelim-07-062.long.html.

[2] J. A. M. Vermaseren, Nucl. Phys. B 229 (1983) 347.

[3] A. Aktas et al. [H1 Collaboration], Eur. Phys. J. C 31 (2003) 17 [hep-ex/0307015].

[4] A. Aktas et al. [H1 Collaboration], Phys. Lett. B 583 (2004) 28 [hep-ex/0311015]. 